\title{
ÍNDICES DE TENTATIVAS DE SUICÍDIO NA FASE DA ADOLESCÊNCIA DEMONSTRA NECESSIDADES PREVENTIVAS NO AMBIENTE DA EDUCAÇÃO
}

\author{
INDICES OF SUICIDE ATTEMPTS IN THE ADOLESCENCE PHASE SHOWS \\ PREVENTIVE NEEDS IN THE EDUCATION ENVIRONMENT
}

\author{
Tiago Moreno Lopes Roberto ${ }^{1}$ \\ Gerardo Maria de Araújo Filho²
}

Elimeire Alves de Oliveira ${ }^{3}$

\section{Ana Paula Rodrigues ${ }^{4}$}

\begin{abstract}
RESUMO: A violência autoprovocada tem ocorrido em diversos perfis epidemiológicos, com variados métodos de tentativas contra a própria vida, caracterizando a tentativa de suicídio, indagando possíveis intervenções para essa população, que se encontra em maior vulnerabilidade psicossocial. Este trabalho visou analisar, por meio de notificações, a caraterização - em um determinado munícipio que se encontra no interior de São Paulo (Votuporanga) - de indivíduos que passaram pelo serviço de saúde após tentar alguma violência contra si mesmo; o período de investigação das ocorrências situa-se entre janeiro de 2015 e junho de 2017. Os dados coletados foram correlacionados de acordo com a localização, sexo, nível escolar, tentativas anteriores, situação conjugal, opção sexual, tipo de tentativa, se possui algum transtorno mental ou deficiência mental, dentre outros aspectos de relevância. Os itens que caracterizam esse indivíduo são preenchidos pelos profissionais da saúde que realizaram o acolhimento da pessoa que tentou o ato contra a própria vida. Os dados epidemiológicos se fizeram essenciais para a compreensão do contexto social e multifatorial encontrados com maior predominância, prevendo possíveis ações biopsicossociais focadas em locais diferentes, onde foi demonstrado que a fase que necessita de maiores cuidados preventivos estão os adolescentes, que apresentaram número expressivo de tentativas de suicídio, visualizando a necessidade de intervenções em escolas e instituições de ensino superior.
\end{abstract}

Palavras-chave: Violência autoprovocada. Perfil epidemiológico. Adolescência.

ABSTRACT: Self-inflicted violence has occurred in several epidemiological profiles, with various methods of trying against one's own life, characterizing the attempted suicide, asking possible interventions for this population, which is at greater psychosocial vulnerability. This work aimed to analyze, by means of notifications, the characterization - in a certain municipality that is in the interior of São Paulo (Votuporanga) - of individuals who went through the health service after trying some violence against themselves; the investigation period for the occurrences is between January 2015 and June 2017. The data collected were correlated according to location, sex, school level, previous attempts, marital status, sexual option, type of attempt, if you have any mental

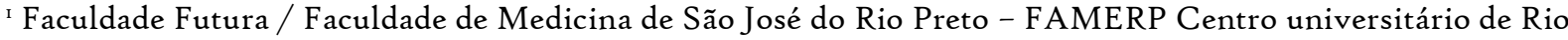
Preto - UNIRP.E-mail: Tiagomorenolopes@hotmail.com.

${ }^{2}$ Faculdade de Medicina de São José do Rio Preto-FAMERP. E-mail: filho.gerardo@gmail.com.

3 Faculdade de Futura. E-mail:Elimeire.alves@gmail.com.

4Faculdade de Faveni /Faculdade Futura. E-mail: diretoria@faveni.edu.br.
} 
disorder or mental deficiency, among other relevant aspects. The items that characterize this individual are filled out by the health professionals who welcomed the person who tried the act against their own lives. Epidemiological data became essential for understanding the social and multifactorial context found with greater predominance, predicting possible biopsychosocial actions focused on different locations, where it was demonstrated that the phase that needs more preventive care are the adolescents, who presented a significant number of attempts suicide, visualizing the need for interventions in schools and higher education institutions.

Keywords: Self-inflicted violence. Epidemiological profile. Adolescence. Keywords: Self-inflicted violence. Epidemiological profile. Adolescence.

\section{INTRODUÇÃO}

Segundo a Organização Mundial de Saúde o ato do suicídio está entre as três principais causas de morte de pessoas entre is a 44 anos de idade, sendo responsável anualmente por um milhão de óbitos (o que corresponde a I,4\% de mortes). Esses dados não incluem as tentativas de suicídio, que são de ro a 20 vezes mais frequentes que o ato do suicídio em si (WHO, 2014).

As taxas de mortalidade por suicídio representam uma pequena parte de problemática das lesões autoprovocadas intencionalmente, gerando um grande número de internações por estas causas que não resultam em óbito. Uma grande parcela destes indivíduos que se lesionam busca atendimento em âmbito ambulatorial, no entanto existem outros que não buscam tratamentos, sejam para as lesões tanto físicas ou psicológicas, e tal situação gera um impacto não apenas no sujeito, mas

em seus familiares, além de refletir no contexto de planejamento de políticas de saúde, no tocante às medidas de prevenção.

Estudo realizado na cidade de Campinas - São Paulo - Brasil, com amostragem de 500 indivíduos, com idade acima de 14 anos e residentes na zona urbana, demonstraram que o sexo feminino tem maior prevalência da ideação suicida, em adultos entre 30 e 40 anos que vivem sozinhos, bem como os espíritas e os de maior renda (BOTEGA et al., 2009).

O Ministério da Saúde, por meio da Portaria $n^{\circ}$ 1.271, de o6 de junho de 2014, ressalta a obrigatoriedade da notificação de violência e agravos para os serviços de saúde, médicos e outros profissionais, serviços públicos ou privados, sendo instrumento fundamental para o conhecimento do perfil da violência, possibilitando intervenções no controle do âmbito epidemiológico e preventivo para o problema de violência (BRASIL, 2014).

Os altos índices de violência, seja ela no âmbito nacional ou internacional, têm gerado um olhar de prioridade e preocupação da saúde pública, envolvendo ações que priorizem o maior envolvimento dos profissionais técnicos da saúde e um maior diálogo com a sociedade, permitindo maiores reflexões sobre a temática violência e suicídio. Trata-se de um fenômeno social que desestrutura um conjunto de elementos biopsicossociais. (GARBIN et al., 2015). 
Ocorreram no Brasil 105.097 internações no SUS (288 casos por dia) nos anos de 2000-2013 decorrentes de lesões autoprovocadas intencionalmente, por indivíduos com idade maior de nove anos, sendo $63.468(60,4 \%)$ do sexo masculino e $4 \mathrm{I} .628(39,6 \%)$ do sexo feminino. Por meio de pesquisas, é possível evidenciar que a sociedade, os familiares e o sistema de saúde podem agir com maior eficácia em modelos de prevenção e atuação profissional, aplicando os procedimentos corretos, disponíveis por meio da OMS (MONTEIRO et al., 2015).

As lesões autoprovocadas são consideradas importante problema de saúde pública, pois constituem um sinalizador de mal-estar e sofrimento de indivíduos, cuja ação geralmente está relacionada a um sentimento de impossibilidade na identificação de alternativas viáveis para a solução de seus conflitos e sofrimentos, optando pelas tentativas de tirar a própria vida como resposta (MONTEIRO et al., 2015).

A presente pesquisa teve como base para elaboração inicial do projeto a observação de profissionais de saúde mental e profissionais da educação que passaram a lidar frequentemente com o fenômeno da violência auto provocada em indivíduos de diversas idades, gerando a busca pela compreensão desse fenômeno que tem afetado as diversas relações, surgindo então a caracterização do indivíduo. Neste contexto, a importância da elaboração de novos projetos nas áreas da saúde e educação podem se revelar medidas importantes de prevenção e por isso devem ser pensados e idealizados por meio da investigação epidemiológica de cada grupo, município ou bairro, revendo questões de maior incidência e prevalência. A inclusão e implementação de novas políticas públicas para esse fenômeno necessitam de um diálogo que desconstrua estigmas de medo e inabilidade dos profissionais.

\section{Metodologia}

2.1 Amostra

Foi redigida uma pesquisa, por meio da coleta de dados no Município de Votuporanga (interior Paulista / Noroeste Paulista), original e bibliográfica, com abordagem quantitativa, baseada em dados descritivos epidemiológicos de violência autoprovocada.

A população ou universo da pesquisa caracterizou-se por ser um conjunto bem definido e com propriedades específicas, no caso, a obtenção dos dados (notificação de violência autoprovocada) de indivíduos que tentaram o ato de autoextermínio no período de janeiro de 2015 há junho de 2017, não havendo critério de exclusão de dados.

A notificação de violência interpessoal/autoprovocada é gerada pelo SINAN (Sistema de Informação de Agravos de Notificação), de plena responsabilidade do departamento de epidemiologia do estado de São Paulo.

Foi solicitada a isenção do TCLE (Termo de consentimento livre esclarecido) da presente pesquisa, tendo como justificativa da ausência do termo que a pesquisa iria analisar os dados de 
notificações de "Violência Interpessoal/AUTOPROVOCADA" no Município de Votuporanga/SP, dados gerados pelo SINAN e que foram disponibilizados por meio de arquivo as descrições epidemiológicas.

\subsection{Materiais}

Foram utilizados os seguintes instrumentos na coleta de dados:

I) Dados digitalizados por meio de anexo em e-mail (enviados pelo departamento da Secretaria de Epidemiologia do Estado de São Paulo).

2) Termos de recebimento do anexo enviado para Secretaria de Epidemiologia do Estado de São Paulo.

\subsection{Procedimento}

A base da investigação se deu início por meio da solicitação dos dados de Violência autoprovocada ao departamento de epidemiologia do município de Votuporanga-SP, os quais foram enviados exclusivamente ao e-mail do pesquisador, por meio arquivo digital. Os dados foram direcionados de acordo com o manual enviado, com a categorização em tabelas no Excel; no arquivo recebido estavam relacionados todos os tipos de violências, como por exemplo: sexual, psicológica dentre outras.

Foram categorizadas e separadas somente as notificações de lesões provenientes de violência autoprovocada, as quais agruparam-se por categorias (tipo de tentativa de suicídio, sexo, idade, nível escolar); em seguida as correlações existentes entre os indivíduos que tentaram o ato de autoextermínio e os dados existentes em outras culturas demográficas, características e métodos utilizados contra a própria vida, além de variáveis demostradas por meio da notificação de violência auto provocada que demonstrassem o perfil epidemiológico do sujeito.

Todos os procedimentos levaram em conta a confidencialidade e a privacidade dos sujeitos e familiares, a fim de que não houvesse estigmatização e nem o uso de suas imagens e informações como forma de prejudicá-los.

\subsection{Análise de dados}

Os dados coletados foram analisados por gráficos de frequência absoluta em Excel. A primeira parte da análise consistiu na exclusão de dados que não faziam parte das lesões e violência autoprovocada. A segunda parte da análise compreendeu o agrupamento de categorias; as propriedades foram descobertas após serem relacionadas com o grau de frequência, chegando assim às respostas e aos objetivos desta pesquisa.

\subsection{Aspectos éticos}

Este estudo foi cadastrado na Plataforma Brasil e encaminhado ao Comitê de Ética e Pesquisa, tendo sido aprovado no dia 17 de julho de 2017, sob parecer de número: 2.175.362 e Certificado de Apresentação para Apreciação Ética (CAAE): 67599917.6.00oo.5415. 


\section{Resultados e discussão}

A notificação de Violência interpessoal/autoprovocada corresponde a fatores externos de morbidade e mortalidade, estabelecido por meio do CID (Código Internacional de Doenças). As lesões podem estar especificadas por envenenamento, enforcamento, afogamento, queimaduras, acidente de trânsito, dentre outros; atualmente, a notificação é o único meio de tabulação para identificação das tentativas de suicídio.

Foram coletados dados epidemiológicos sobre a violência autoprovocada no município de Votuporanga-SP no período de janeiro de 2015 a junho de 2017, nos quais 234 notificações foram caracterizadas como intoxicação exógena e 26 notificações especificadas como tentativa de suicídio, conforme Figura I.

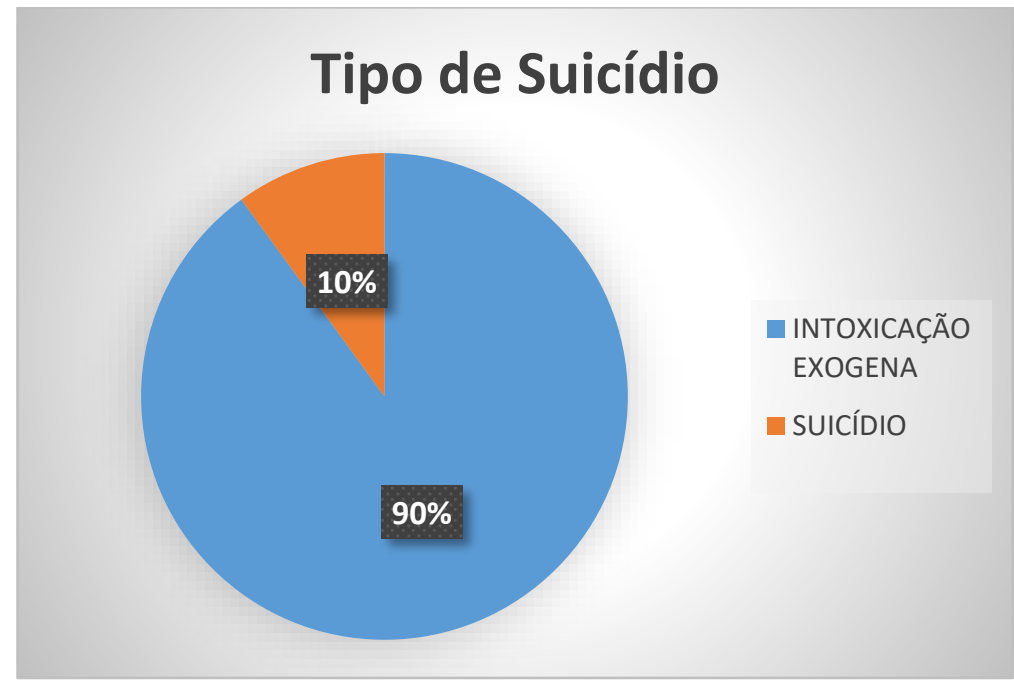

\section{Categoria: Tipo de violência autoprovocada}

Fonte: SINAN, 2015.

Observou-se que o comportamento mais frequente na população de Votuporanga-SP foi a intoxicação exógena; no entanto, analisando as notificações foi possível encontrar falhas de preenchimento na especificação da violência contra si, além de ausência da categorização do tipo de intoxicação (se ingestão de veneno, medicação, chá, dentre outros que poderiam ter sido utilizados no procedimento). Por conta dessas omissões caracterizou-se o despreparo do profissional da saúde, que não está atento a esses detalhes que são fundamentais nos métodos preventivos e para o surgimento de políticas públicas.

Uma pesquisa realizada no Rio de Janeiro fez uma análise das tentativas e dos suicídios por intoxicação exógena e notou-se que o preenchimento da notificação não continha informações básicas como: endereço completo data de nascimento, nome da mãe, e que alguns casos de suicídio não haviam sequer sido registrados, porém haviam sido relatados por médicos. Os dados do estudo 
concluíram que é possível melhorar as informações das subnotificações de fenômenos relevantes e pouco estudados, e que a pesquisa se deparou com dificuldades em identificar adequadamente intoxicação como intencional ou não intencional. O problema que nem sempre é de difícil deteç̧ão e correção, soma-se a tão conhecida "subnotificação de tentativa/suicídio" por motivos socioculturais e econômicos. Além dos erros encontrados nas classificações, a pesquisa ressaltou algumas implicações legais relacionadas à notificação do SINAN, e ao descaso dos profissionais com a portaria GM/MS no I04/20II, que estabelece as responsabilidades e atribuições dos profissionais e serviços de saúde determinando seguir suas obrigatoriedades (SANTOS et al., 2014).

A categoria suicídio foi acrescentada na notificação onde o profissional da saúde descreveu como tal, porém não especificou a forma da tentativa, permitindo até mesmo uma reflexão sobre a visão que os profissionais da saúde têm em relação ao comportamento suicida, que é considerado como "Não querer viver". Então, quais métodos de violência são considerados como ato de suicídio? Por meio das notificações nota-se a ausência de habilidade e capacitação do profissional ao redigir a notificação, o que leva a repensar as práticas de capacitação dos profissionais da rede de urgência e emergência e do diálogo com a rede de saúde mental.

Os dados coletados em Votuporanga-SP demonstraram que não há outras formas de tentativas de suicídio como enforcamento, objeto cortante, objeto quente entre outros métodos que a notificação oferece como opção para detalhamento e melhor compreensão do evento relatados no período pesquisado. Notadas ausências de relatos de tentativas consideradas como fenômenos mais "comuns" dentro do comportamento suicida, o pesquisador perguntou ao coordenador da rede de saúde mental quais seriam mais recorrentes, e foi informado que a tentativa de auto mutilação é um dos comportamentos que ocorre com maior frequência no munícipio; porém, não foram encontradas notificações detalhadas com essa categoria, demonstrando possíveis erros ou omissão dos fenômenos.

Em outras pesquisas publicadas no contexto brasileiro, a intoxicação exógena aparece como a categoria de maior incidência nas tentativas de suicídio e violência autoprovocada, e indivíduos do sexo feminino figuram como predominantes na escolha desse método. Os profissionais da rede de saúde do munícipio de Votuporanga não categorizaram ou detalharam nas notificações os fenômenos como enforcamento, quedas, objeto perfurante, entre outros meios de violência que configuram tentativas de suicídio; sendo assim, os dados não retratam fielmente o cenário epidemiológico dos municípios brasileiros.

\section{Categoria: Sexo}

A figura 2 apresenta o sexo dos indivíduos que tentaram a violência autoprovocada, sendo a maior prevalência do sexo feminino com 2ı notificações, contra 50 do sexo masculino. 


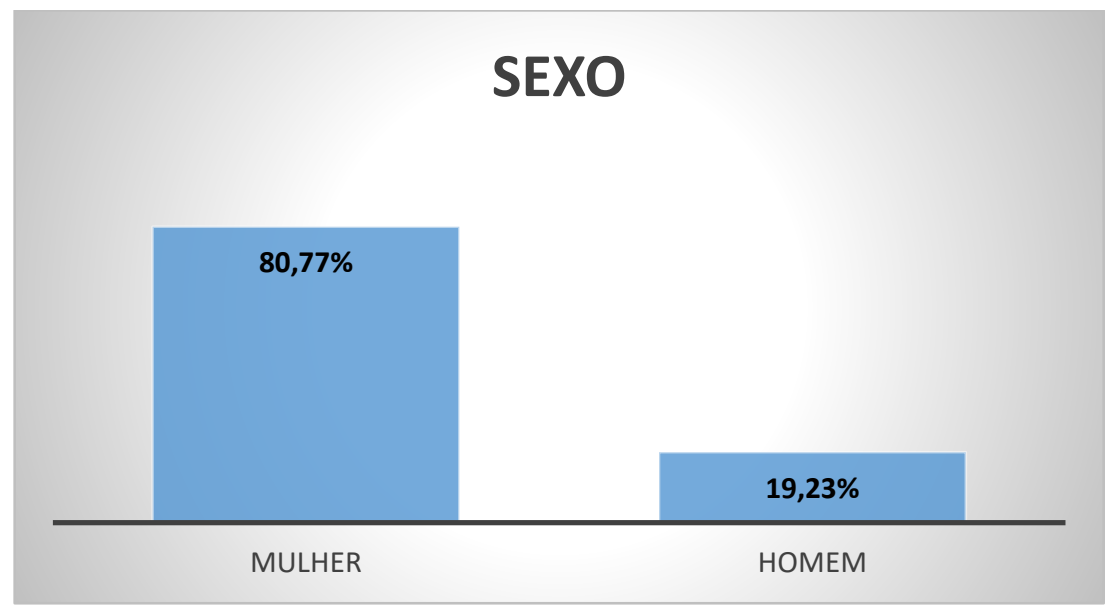

Figura 2 - Sexo dos indivíduos que tentaram a violência autoprovocada Fonte: SINAN, 20I5.

Pesquisa realizada no âmbito brasileiro sobre a caracterização de tentativas por substâncias exógenas demonstrou a variância entre sexo masculino e feminino, com maior predominância envolvendo o feminino em função dos agentes tóxicos utilizados; quando analisada a faixa etária pode-se comparar que os adultos $(46,7 \%)$ e adolescentes $(72,7 \%)$ utilizaram predominantemente o medicamento, e os idosos (43,7\%), principalmente agrotóxicos. Pelos resultados obtidos verificou-se que o suicídio foi frequente entre os homens que fizeram uso de agrotóxicos. $\mathrm{O}$ fácil acesso às substâncias tóxicas, demonstra favorecer o impulso de tentar o suicídio, e pensar em medidas protetivas e de conscientização sobre alguns comportamentos visíveis do trabalhador é de extrema importância, bem como ações de controle da venda de agrotóxicos que acolham e promovam assistência adequada aos suicidas, por meio de campanhas e informativos no ato da venda. (VIEIRA; SANTANA; SUCHARA, 2015).

\section{Categoria: Faixa Etária}

A figura 3 apresenta a faixa etária dos indivíduos que tentaram o ato de violência autoprovocada, estando os de 26 a 30 anos em primeiro, com maior número de indivíduos e 74 notificações, em segundo os de I6 a 25 anos com 68 notificações, os de 35 a 46 anos com 5 I notificações. Os indivíduos com mais de 47 anos foram responsáveis por 44 notificações e aqueles com menos de 13 anos com 23 notificações.

De acordo com os dados brasileiros epidemiológicos correlacionados com o número de óbitos por suicídio no Brasil percebe-se que estes estão voltados a faixa etária de 30 a 40 anos; apenas a raça indígena apresenta um índice elevado na idade de Io a 19 anos, sendo o índice mais alto de óbitos por suicídio no Brasil.

Observa-se um índice expressivo de tentativas na fase de transição de adolescente para a vida adulta na pesquisa do município de Votuporanga, destacando a idade de 16 a 25 anos. De acordo com 
o perfil epidemiológico Brasileiro, apenas a população indígena tem maior risco pela alta proporção de suicídio consumado de adolescentes; os dados de Votuporanga demonstram um número expressivo de tentativas na fase da adolescência. Estes dados são concordantes com várias regiões do mundo, indicando um grau de vulnerabilidade dessa população, exaltando os altos índices de suicídio por meio de consumo de substâncias psicoativas nos jovens indígenas e demais regiões como o Alaska, o Canadá, a Groenlândia, os países nórdicos e a Rússia. (BRASIL, 2017).

Identificar os fatores de risco ao suicídio na adolescência é de extrema importância para prevenção; nesse sentido, todos os profissionais devem ter um olhar mais amplo para interpretá-los e auxiliar de forma adequada; na fase da adolescência identificar os fatores de risco se torna insuficiente para que seja possível evitar o suicídio, é necessário considerar os diversos fatores de risco e exposição, pois muitos adolescentes não desencadeiam pensamentos de morte ou ideações e a ausência dos sinais e fatores de risco ao suicídio não impede que um adolescente possa vir a tentar ou a cometer o ato do suicídio (BRAGA; DELL'AGLIO, 2013).

Os fatores de maior predominância a risco nas tentativas na fase da adolescência são: sofrer violência física e abuso sexual, sofrer ameaças de colegas ou algum tipo de violência psicológica, ter depressão, ter problemas de identidade de gênero, vivenciar relacionamentos não correspondidos, isolamento social, dificuldades de desempenho escolar e aprendizagem, dificuldades para se comunicar com professores, colegas ou familiares. O método mais utilizado por adolescentes para a tentativa de suicídio tem ocorrido por meio de ingestão de medicamentos e considera-se a idade de maior risco como sendo a de $\mathrm{I} 4$ anos (PCRJ/SMS, 2016).

Em pesquisa realizada no âmbito brasileiro sobre ideação suicida em adolescentes do ensino médio, pode-se observar que dos 22,2\% com ideação suicida, $55 \%$ são do sexo feminino e encontramse na faixa etária entre 17 e 19 anos, $45 \%$ cursam o $2^{2}$ ano do ensino médio, $95 \%$ são solteiros; $85 \%$ moram com os pais; 70\% afirmam ser católicos e I0o\% disseram não possuir vinculo ou trabalho remunerado (ARAÚJO; VIEIRA; COUTINHO, 2010).

No processo de adolescer pode ocorrer uma grande busca por soluções imediatas demonstrando comportamentos agressivos e suicidas. Tais comportamentos na fase adolescente vêm como um pedido expressivo de ajuda frente a um intenso sofrimento e/ou dor psicológica insuportável; a depressão é o transtorno mental mais comum associado a ideação suicida com comportamentos agressivos e com o uso de substâncias psicoativas entre os adolescentes (OLIVEIRA et al., 2017).

Por meio dos dados de Votuporanga, a idade de 16 a 25 é a segunda maior frequência de tentativas de suicídio, direcionando maior atenção aos fatores sociais de exposição, início da vida afetiva e das relações de trabalho, dentre outros aspectos, o que exige um olhar específico e qualificado para tomada de medidas preventivas em abrangência escolar e cursos universitários. 
Desta forma, direcionar políticas públicas e meios expressivos de diálogo entre educadores e profissionais da saúde é um meio de ampliar a atenção a essa população adolescente.

Direcionar educação em saúde em especial para os professores e seus alunos é fundamental, já que é possível identificar muitos mitos e tabus sobre o ato suicida e estimular as buscas pelo conhecimento e promoção da saúde mental. No período escolar existem fatores propícios aos riscos como bullying, desestruturação e cargas elevadas de estresse podem influenciar no desenvolvimento suicida; desta forma, é de suma importância que os educadores saibam reconhecer logo no início os sinais de ideação suicida, tornando possível a realização de medidas preventivas (SANTOS et al., 2014).

Torna-se imperiosa a implementação de protocolos de atendimento no serviço de emergência que visem o diálogo dos serviços de saúde mental direcionando o adolescente que tentou o ato de suicídio para acompanhamento psiquiátrico e até mesmo visitas no âmbito escolar e domiciliar para compreensão do seu contexto social e do seu desenvolvimento.

Os índices da fase adulta de 26 a 35 anos demonstra ainda um adiantamento de idade na busca pelo suicídio, a desmotivação precoce está associada à qualidade de vida, e fatores laborais, sendo uma das fases mais produtivas do ciclo da vida, na qual os ideais e planejamento estrutural da vida afetiva e econômica têm maior ênfase.

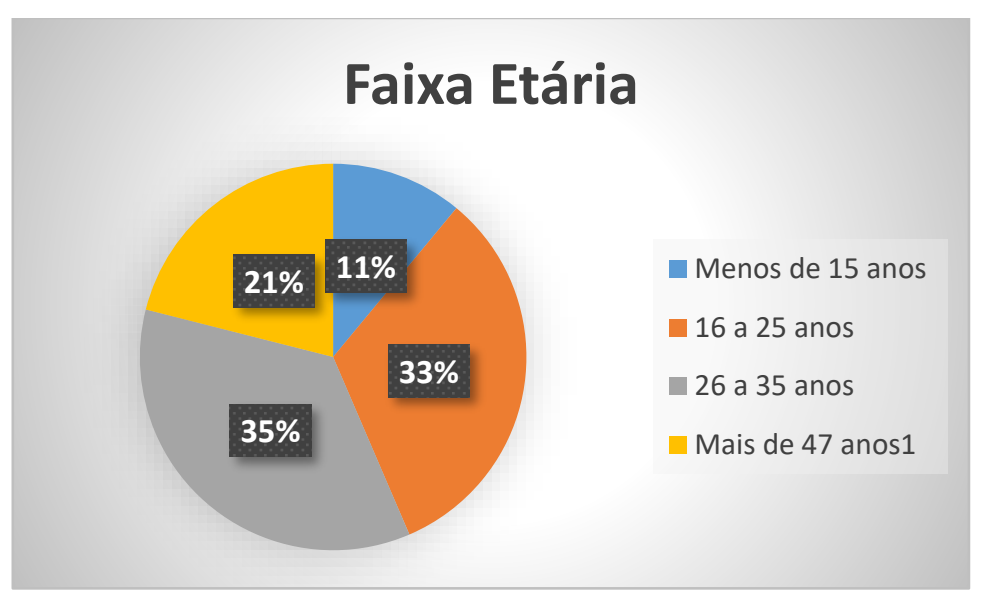

Figura 3 - Faixa Etária por frequência de notificações de violência autoprovocada Fonte: SINAN, 2015.

\section{Categoria: Nível Escolar}

$\mathrm{Na}$ figura 4 observa-se que os indivíduos que mais tentaram a violência autoprovocada foram aqueles do nível de ensino médio e, e em segundo lugar, os indivíduos que estudaram da $5^{\circ}$ a $8^{\circ}$ série.

No município da pesquisa, o maior número de tentativas de suicídio foi com o nível escolar ensino médio, demonstrando e se relacionando com a fase da adolescência, além de pessoas que não deram continuidade nos estudos de nível superior. 
É possível, neste aspecto, afirmar que os dados obtidos se relacionam com o do contexto brasileiro, que apresenta $30,1 \%$ dos que tentaram contra a própria vida com escolaridade de ensino fundamental incompleto ou completo e $22,6 \%$ ensino médio incompleto ou completo (BRASIL, 2017).

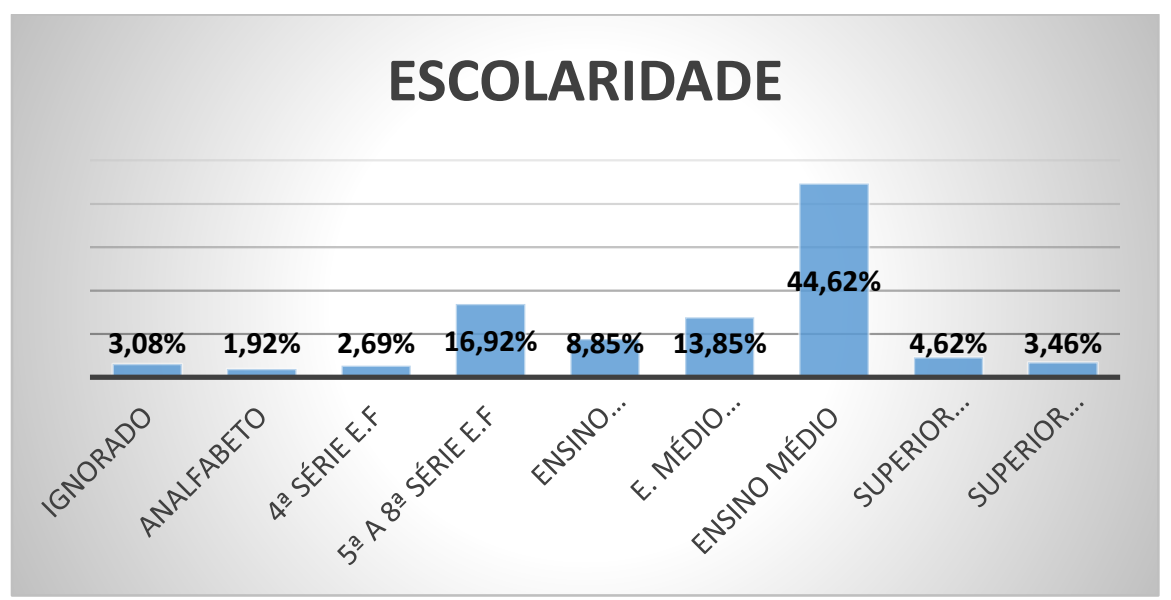

Figura 4 - Escolaridade dos indivíduos que tentaram o ato de violência autoprovocada Fonte: SINAN, 2015.

\section{Conclusões}

Os resultados obtidos por meio da análise das notificações de violência autoprovocada no município de Votuporanga-SP demostraram inicialmente que o perfil dos indivíduos que realizaram tentativa de suicídio com maior frequência foi o sexo feminino com $80,77 \%$, seguido do sexo masculino, com 19,23\%, sendo de maior prevalência no nível escolar médio. Em relação ao grupo populacional constatou-se que 35\% das tentativas estão na faixa etária de 26 a 35 anos e 33\% das tentativas com a faixa etária de I6 a 25 anos, demonstrando uma alta elevação na fase da adolescência. O método intoxicação exógena foi responsável por 90\% das notificações e outros 10\% foram relatados de fato como suicídio não havendo outros métodos.

Traçar um perfil do indivíduo que pratica a violência autoprovocada, a tentativa de suicídio ou que sofre o suicídio consumado não é fácil. $\mathrm{O}$ assunto ainda está fragmentado e carrega o estigma do preconceito e desprezo, tanto nos setores da saúde quanto nas relações sociais e culturais. Por isso, criar e desenvolver estratégias preventivas apenas na área da saúde é uma tarefa muito árdua, pois há casos que nem chegam ao conhecimento dos profissionais da saúde, por isso a importância de um trabalho integrado às outras instituições, em especial à educação, tendo em vista que os dados epidemiológicos obtidos permitiram revelar que a população que apresenta maior fragilidade são os estudantes, em específico os adolescentes.

Ou seja, se os sujeitos que evidenciam maior potencial para a violência autoprovocada fazem parte de um grupo que está, até mesmo por força de lei, inserido em instituições estabelecidas 
socialmente, que são as escolas, é de grande importância ações que privilegiem o trabalho em rede, frutos de parcerias que trarão pistas para o enfrentamento desse problema. Neste sentido, o trabalho integrado que estabeleça canais eficazes de comunicação entre os órgãos da saúde e da educação, que ultrapassaria paradigmas calcados no trabalho verticalizado e restrito.

Desta forma, o conceito de saúde deve se estender para as escolas e universidades, onde de um lado seria possível capacitar os profissionais da educação, que lidam diretamente com esse segmento populacional, a desenvolver um olhar direcionado para o sofrimento psíquico e ao mesmo tempo desenvolver estratégias de comunicação entre rede da educação e rede de saúde para minimizar a ocorrência de atos autodestrutivos contra a vida, como é o caso da violência autoprovocada.

\section{Referências}

ARAÚJO, L. C.; VIEIRA, K. F. L.; COUTINHO, M. P. L. Ideação suicida na adolescência: um enfoque psicossociológico no contexto do ensino médio. Psico-USF, v. I5, n. I, p. 47-57, jan./abr., 2010.

BOTEGA, N. J. et al. Prevalências de ideação, plano e tentativa de suicídio: Um inquérito de base populacional em Campinas, São Paulo, Brasil. Cad. Saúde Pública, v. 25, n. 12, p. 2632-2638, 2009.

BRAGA, L. L.; DELL'AGLIO, D. D. Suicídio na adolescência: fatores de risco, depressão e gênero.

Contextos Clínicos, v. 6, n. I, p. 2-14, jan.-jun., 2013.

BRASIL. Ministério da Saúde. Portaria no 1.271, de 6 de junho de 2014. Define a Lista Nacional de Notificação Compulsória de doenças, agravos e eventos de saúde pública nos serviços de saúde públicos e privados em todo o território nacional, nos termos do anexo, e dá outras providências. Disponível em: http://bvsms.saude.gov.br/bvs/saude legis/gm/2014/prt 1271_06_o6_2014.html. Acesso em: 15 jul. 2020 .

Secretaria de Vigilância em Saúde. Ministério da Saúde. Perfil epidemiológico das tentativas e óbitos por suicídio no Brasil e a rede de atenção à saúde. Boletim Epidemiológico, v. 48, n. 30, p. I-I4, 2017.

GARBIN, C. A. S. et al. Desafios do profissional de saúde na notificação da violência: obrigatoriedade, efetivação e encaminhamento. Ciência \& Saúde Coletiva, v. 20, n. 6, p. 1879-189o, 2015 .

MONTEIRO, R. A. et al. Hospitalizações relacionadas a lesões autoprovocadas intencionalmente Brasil, 2002 a 2013. Ciência \& Saúde Coletiva, v. 20, n. 3, p. 689-70o, 2015.

OLIVEIRA, M. A. et. al. Comportamento suicida entre adolescentes: Revisão integrativa da literatura nacional. Adolesc. Saude, v. I4, n. I, p. 88-96, jan./mar., 2017. 
PCRJ - Prefeitura da Cidade do Rio de Janeiro / SMS - Secretaria Municipal de Saúde. Superintendência de Atenção Primária. Avaliação do risco de suicídio e sua prevenção. 2016. Disponível em: https://subpav.org/download/prot/Guia_Suicidio.pdf. Acesso em: I5 jul. 2020.

SANTOS, S. A. et al. Tentativas e suicídios por intoxicação exógena no Rio de Janeiro, Brasil: análise das informações através do linkage probabilístico. Cad. Saúde Pública, v. 30, n. 5, p. 1057Io66, mai., 2014.

SINAN - Sistema de Informação de Agravos e Notificação. Violência interpessoal / autoprovocada - Votuporanga-SP. Disponível em: http://webcache.googleusercontent. $\mathrm{com} /$ search?q=cache:rkAPmBmzfdMJ:portalsinan.saude.gov.br/violencia-interpessoalautoprovocada $+\& c d=\mathrm{I} \& \mathrm{hl}=\mathrm{pt}-\mathrm{BR} \& \mathrm{ct}=\mathrm{clnk} \& \mathrm{gl}=\mathrm{br}$. Acesso em: is jul. 2020.

VIEIRA, L. P.; SANTANA, V. T. P.; SUCHARA, E. A. Caracterização de tentativas de suicídios por substâncias exógenas. Cad. Saúde Colet., v. 23, n. 2, p. II8-123, 2015.

WHO - Word Health Organization. Country reports and charts available. 2014. Disponível em: http://www.suicidepreventionhelp.com/directory/detail/link-I479.html. Acesso em: I5 jul. 2020. 